Pacific Journal of Mathematics

ON THE FIELD OF RATIONAL FUNCTIONS OF ALGEBRAIC 


\section{ON THE FIELD OF RATIONAL FUNCTIONS OF ALGEBRAIC GROUPS}

\section{A. Bialynicki-Birula}

o. Introduction. Let $K$ be an algebraically closed field of characteristic 0 , let $k$ be a subfield of $K$ and suppose that $G$ is a $(k, K)$ algebraic group, i.e., an algebraic group defined over $k$ and composed of $K$-rational points. Let $k(G)$ denote the fields of $k$-rational functions on $G$. $\quad G_{k}$ denotes the subgroup of $G$ composed of all $k$-rational points of $G$. If $g \in G_{k}$ then the regular mapping $L_{g}\left(R_{g}\right)$ of $G$ onto $G$ defined by $L_{0} x=g x\left(R_{0} x=x g\right)$ induces an automorphism of $k(G)$ denoted by $g_{l}\left(g_{r}\right)$. Let $D_{k}$ denote the Lie algebra of all $k$-derivations of $k(G)$ (i.e., of all derivations of $k(G)$ that are trivial on $k$ ) which commute with $g_{r}$, for every $g \in G_{k}$.

For any subset $A$ of $k(G)$ let $G(A)$ denote the subgroup of $G$ composed of all elements $g$ such that $g_{r}(f)=f$, for every $f \in A$. In the sequel we shall always assume that $G_{k}$ is dense in $G$.

The main result of this paper is the following theorem:

THEOREM 1. Let $F$ be a subfield of $k(G)$ containing $k$. Then the following three conditions are equivalent:

(1) $F$ is $\left(G_{k}\right)_{l}$ - stable

(2) $F$ is $D_{k}$ - stable

(3) $F=k(G / G(F))$ and so $F$ coincides with the field of all elements of $k(G)$ that are fixed under $G(F)_{r}$.

By means of the theorem, we establish a Galois correspondence between a family of subgroups of $G$ and the family of $\left(G_{k}\right)_{i}$-stable subalgebras of the algebra of representative functions of $G$.

The author wishes to express his thanks to Professor G.P. Hochschild and Professor M. Rosenlicht for a number of instructive conversations on the subject of this note.

1. Let $K$ be an algebraically closed field of characteristic 0 , let $k$ be a subfield of $K$ and suppose that $V, W$ are $(k, K)$ - algebraic varieties. Let $k(V), k(W)$ denote the fields of $k$-rational functions on $V$ and $W$, respectively. If $A$ is a subset of $k(V)$ then $k(A)$ denotes the fields generated by $k$ and $A$.

The following result is known ${ }^{1}$ :

(1) Let $F$ be a rational mapping of $V$ onto a dense subset of $W$ and let $\varphi$ be the cohomomorphism corresponding to $F$. Then there exists

Received September 28, 1960, in revised form November 14, 1960.

1 See e.g. [2], 
an open subset $W_{1} \subset W$ such that $F^{-1}(x)$ contains exactly [k(V): $\left.\varphi(k(W))\right]$ elements, for every $x \in W_{1}$.

LEMma 1. Let $A$ be a subset of $k(V)$ and suppose that there exists $a$ dense set $V_{1} \subset V$ and an open subset $V_{2} \subset V$ such that for any two distinct points $x_{1}, x_{2}$, where $x_{1} \in V_{1}, x_{2} \in V_{2}$, there exists a function $f \in A$ which is defined at $x_{1}, x_{2}$ and $f\left(x_{1}\right) \neq f\left(x_{2}\right)$. Then $k(A)=k(V)$.

Proof. Let $B$ be a finite subset of $A$, say $B=\left\{f_{1}, \cdots, f_{n}\right\}$. Then $F_{B}$ denotes the rational mapping $F_{B}: V \rightarrow K^{n}$ defined by $F_{B}(x)=\left(f_{1}(x)\right.$, $\left.\cdots, f_{n}(x)\right)$ and $W_{B}=\left(F_{B}(V)^{-} \subset K^{n}\right.$. Let $\Delta\left(W_{B}\right)$ be the diagonal of $W_{B} \times W_{B}$ and $V_{B}=\left(\left(F_{B} \times F_{B}\right)^{-1} \Delta\left(W_{B}\right)\right)^{-} \subset V \times V$. Then there exists a finite subset $B_{0} \subset A$ such that $V_{B_{0}} \subset V_{B}$, for every finite subset $B \subset A$ (since $V \times V$ satisfies the minimal condition for closed sets). Let $V_{0}$ be an open subset of $V$ such that $F_{B_{0}}$ is regular on $V_{0}$. We may assume that $V_{0}=V_{2}=V$, since we may replace $V$ by $V_{0} \cap V_{2}$. If $x_{1} \in V_{1}, x_{2} \in V$ and $x_{1} \neq x_{2}$ then there exists $f \in A$ such that $f$ is defined at $x_{1}, x_{2}$ and $f\left(x_{1}\right) \neq f\left(x_{2}\right)$. Hence $\left(x_{1}, x_{2}\right) \notin V_{\{f\}}$ and so $\left(x_{1}, x_{2}\right) \notin V_{B_{0}}$. Thus $F_{B_{0}}\left(x_{1}\right) \neq F_{B_{0}}\left(x_{2}\right)$. Therefore, for every $x \in F_{B_{0}}\left(V_{1}\right), F_{B_{0}}^{-1}(x)$ contains exactly one element. But $F_{B_{0}}\left(V_{1}\right)$ is dense in $W_{B_{0}}$. Hence it follows from (i ) that $\left[k(V): k\left(B_{0}\right)\right]=1$, i.e., $k(V)=k\left(B_{0}\right)$. Thus $k(V)=k(A)$.

Let $G$ be a $(k, K)$ - algebraic group. Suppose that $G_{k}$ is dense in $G$. Let $D$ be the Lie algebra of all derivations of $K(G)$ commuting with $g_{r}$, for every $g \in G$, and let $D_{k}$ denote the Lie algebra consisting of all derivations from $D$ that map $k(G)$ into $k(G)$. Let $k[D](K[D])$ denote the $k$-algebra ( $K$ - algebra) of transformations generated by the identity map and $D_{k}(D)$.

If $d \in D_{k}$ then $d$ restricted to $k(G)$ is a $k$-derivation commuting with $g_{r}$, for every $g \in G_{k}$. On the other hand if $d_{1}$ is a $k$-derivation of $k(G)$ commuting with $g_{r}$, for every $g \in G_{k}$, then there exists a unique extension $d$ of $d_{1}$ to a $K$-derivation of $K(G)$, and the extension belongs to $D_{k}$. Hence we may identify $D_{k}$ and the Lie algebra of all $k$-derivations of $k(G)$ that commute with $g_{r}$, for every $g \in G_{k}$.

(ii) ${ }^{2}$ If $f \in K(G)$ and $f$ is defined at a point $g \in G$ then $d f$ is defined at $g$, for any $d \in K[D]$.

Lemma 2. Let $f \in K(G)$ and suppose that $f$ is defined at $g \in G_{k}$. If $f \neq 0$ then there exists $d \in k[D]$ such that $(d f)(g) \neq 0$.

Proof. Suppose that $f \neq 0$. If $f(g) \neq 0$ then the identity element of $k[D]$ satisfies the desired condition. Hence we may assume that $f(g)=0$, Let $\mathscr{O}_{k}\left(\mathcal{O}_{K}\right)$ denote the local ring of $g$ in $k(G)(K(G))$ and let $m_{k}\left(m_{K}\right)$ be the maximal ideal of $\mathscr{O}_{k}\left(\mathscr{O}_{K}\right)$. Then $f \in m_{K}$. Let 
$x_{1}, \cdots, x_{m}$ be elements of $m_{k}$ such that $x_{1}+m_{k}^{2}, \cdots, x_{m}+m_{k}^{2}$ is a $k$ basis of $m_{k} / m_{k}^{2}$. The $x_{1}+m_{K}^{2}, \cdots, x_{m}+m_{K}^{2}$ is a $K$-basis of $m_{K} / m_{K}^{2}$. Hence every mapping $\left(x_{1}, \cdots, x_{m}\right) \rightarrow k$ can be extended to a derivation $\partial: \mathscr{O}_{K} \rightarrow K$. On the other hand $f \neq 0$ and so there exists an integer $t$ such the $f \in m_{K}^{t}-m_{K}^{t+1}$. Hence $f=\sum_{i_{1}+\cdots+i_{m}=t} a_{i_{1}, \ldots, i_{m}} x_{1}^{i_{1}}, \cdots, x_{m}^{i_{m}}+f_{1}$, where $f_{1} \in m_{K}^{t+1}, a_{i_{1}, \ldots, i_{m}} \in K$ and at least one $a_{i_{1}, \ldots, i_{m}}$ is different from zero. Let $\partial_{i}$ be the derivation of $\mathcal{O}_{K}$ into $K$ such than $\partial_{i} x_{j}=\delta_{i j}$, where $\delta_{i j}=\left\{\begin{array}{l}0 \text { if } i \neq j \\ 1 \text { if } i=j .\end{array}\right.$ It is known ${ }^{3}$, that there exist $d_{i} \in D_{k}$ such that $\left(d_{i} f\right)(g)=$ $\partial_{i} f$ for every $f \in \mathcal{O}_{K}$. Then $\left(d_{1}^{i_{1}} \cdots d_{m}^{i_{m}}\right) f(g)=i_{1} ! \cdots i_{m} ! a_{i_{1}, \ldots, i_{m}} \neq 0$ if $a_{i_{1}, \ldots, i_{m}} \neq 0$. Hence the lemma is proved.

If $A$ is a subset of $k(G)$ then $G(A)$ denotes the subgroup of $G$ composed of all elements $g$ such that $g_{r}$ leaves the elements of $A$ fixed. For any $A \subset k(G), G(A)$ is a $k$-closed subgroup of $G$.

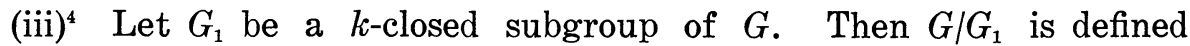
over $k$. Let $\varphi$ be the cohomomorphism of $k\left(G / G_{1}\right)$ into $k(G)$ corresponding to the canonical mapping $G \rightarrow G / G_{1}$. Then $\rho\left(k\left(G / G_{1}\right)\right)$ coincides with the subfield of all elements of $k(G)$ which are fixed under $g_{r}$, for every $g \in G_{1}$. In the sequel we shall identify $k\left(G / G_{1}\right)$ and $\varphi\left(k\left(G / G_{1}\right)\right)$.

\section{Proof of the theorem.}

Implications $(3) \Rightarrow(1)$ and $(3) \Rightarrow(2)$ are obvious.

$(1) \Rightarrow(3)^{5}$. Let $g_{1} \in G_{k}, g_{2} \in G$ and $G(F) g_{1} \neq G(F) g_{2}$. Then $g_{2} g_{1}^{-1} \notin G(F)$. Hence there exists $f_{0} \in F$ such that $\left(g_{2} g_{1}^{-1}\right)_{r} f_{0} \neq f_{0}$. Therefore there exists an element $g \in G_{k}$ such that $\left(g_{2} g_{1}^{-1}\right)_{r} f_{0}$ and $f_{0}$ are defined at $g$ and $\left(g_{2} g_{1}^{-1}\right)_{r} f_{0}(g) \neq f_{0}(g)$, i.e., $f_{0}\left(g_{2} g_{1}^{-1} g\right) \neq f_{0}(g),\left(g_{1}^{-1} g\right)_{l} f_{0}\left(g_{2}\right) \neq\left(g_{1}^{-1} g\right)_{l} f_{0}\left(g_{1}\right)$. Let $f=\left(g_{1}^{-1} g\right)_{l} f_{0}$. Then $f \in F$ since $g_{1}^{-1} g \in G_{k} ; f$ is defined at $g_{1}$ and $g_{2}$, and $f\left(g_{1}\right) \neq f\left(g_{2}\right)$. Thus it follows from Lemma 1 that $F=k(G / G(F))$, because $G(F) \cdot G_{k} / G(F)$ is dense in $G / G(F)$.

$(2) \Rightarrow(3)$. Let $f_{1}, \cdots, f_{n}$ be a set of generators of $F$ over $k$, and let $V_{1}$ be an open subset of $G$ such that $f_{1}, \cdots, f_{n}$ are regular on $V_{1}$. We may assume that $V_{1}=G(F) V_{1}$. Let $g_{1} \in V_{1} \cap G_{k}, g_{2} \in V_{1}, G(F) g_{1} \neq$ $G(F) g_{2}$. Then $g_{2} g_{1}^{-1} \notin G(F)$ and so there exists $f_{i}$ such that $\left(g_{2} g_{1}^{-1}\right)_{r} f_{i} \neq f_{i}$. We know that $\left(g_{2} g_{1}^{-1}\right)_{r} f_{i}$ and $f_{i}$ are defined at $g_{1}$. Hence it follows from Lemma 2 that there exists an element $d \in k[D]$ such that

$$
d\left(\left(g_{2} g_{1}^{-1}\right)_{r} f_{i}\right)(g) \neq\left(d f_{i}\right)(g) \text {, i.e., }\left(d f_{i}\right)\left(g_{1}\right) \neq\left(d f_{i}\right)\left(g_{2}\right) \text {. }
$$

Therefore, for any pair of distinct elements $G(F) g_{1}, G(F) g_{2}$ such that

$$
G(F) g_{1} \in G(F) \cdot G_{k} \cap V_{1} / G(F) \text { and } G(F) g_{2} \in V_{1} / G(F),
$$

3 See [4] p. 51 ,

${ }^{4}$ See Proposition 2, p. 495 in [5].

5 This part of the proof is modeled after the proof of Lemma 5.3 p. 515 in [3]. 
there exists an element $f \in F$ which is defined at $G(F) g_{1}, G(F) g_{2}$ and such that $f\left(G(F) g_{1}\right) \neq f\left(G(F) g_{2}\right)$. But $V_{1} / G(F)$ is an open subset of $G / G(F)$, and $G(F) G_{k} \cap V_{1} / G(F)$ is dense in $G / G(F)$. Hence it follows from Lemma 1 that $F=k(G / G(F))$.

This completes the proof of the theorem.

2. Applications. As a consequence of Lemma 2 one can get the following corollary:

CoROllaRY. If $\alpha$ is an automorphism of $k(G)$ commuting with $D_{k}$ and leaving the elements of $k$ fixed then there exists $h \in G_{k}$ such that $\alpha=h_{r}$.

Proof. $\alpha$ induces a rational map $F_{\alpha}: G \rightarrow G$. Let $g \in G_{k}$ be a point such that $F_{\alpha}$ is defined at $g$ and let $F_{\alpha}(g)=h^{-1} g$ Then $h \in G_{k}$ and $f(g)=(\alpha f)\left(h^{-1} g\right)$, for every $f \in k(G)$ that is defined at $g$. Hence $(d f) g=$ $(\alpha(d f))\left(h^{-1} g\right)$, for every $d \in k[D]$. But $(\alpha(d f))\left(h^{-1} g\right)=\left(h_{r}^{-1}(\alpha(d f))\right)(g)$ and $d$ commutes with $\alpha$ and $h_{r}^{-1}$. Therefore $(d f)(g)=\left(d\left(h_{r}^{-1}(\alpha f)(g)\right)\right)$. Hence it follows from Lemma 2 that $f=h_{r}^{-1}(\alpha f)$. Thus $h_{r} f=\alpha f$, for every $f$ that is defined at $g$. Therefore $h_{r} f=\alpha f$, for every $f \in k(G)$.

It follows from the corollary that if $F$ is a $D_{k}$ - stable subfield of $k(G)$ containing $k$ then every $D_{k}$ - automorphism of $k(G)$ leaving the elements of $F$ fixed belongs to $G(F)_{r}$, i.e., the $D_{k}$ - Galois group of $k(G)$ over $F$ coincides with $G(F)_{r}$. Combining this result and the above theorem we obtain that there exists the usual one to one Galois correspondence between $D_{k}$ - stable subfields of $k(G)$ containing $k$ and $k$-closed subgroups of $G$.

Let $k[G]$ denote the ring of regular (i.e., representative) functions on $G$. Let $\mathscr{R}$ be the family of all $\left(G_{k}\right)_{l}$ - stable (or, equivalently, $D_{k}-$ stable) subrings $R$ of $k[G]$ containing $k$ and satisfying the following condition if $f \in R, g \in R$ and $f / g \in k[G]$ then $f / g \in R$. Let $\mathscr{G}$ denote the family of all $k$-closed subgroups $H$ of $G$ such that $G / H$ is isomorphic to an open subset of an affine variety.

THEOREM 2. The mappings $H \rightarrow k[G] \cap k(G / H)$ and $R \rightarrow G(R)$ establish a Golois correspondence between $\mathscr{G}$ and $\mathscr{R}^{6}$.

Proof. $H \in \mathscr{G}$ then $k[G] \cap k(G / H) \in \mathscr{R}$ and $G(k[G] \cap k(G / H))=H$, since $k(G / H)$ is generated by $k[G] \cap k(G / H)$.

Now, if $R \in \mathscr{R}$ then $G(R) \in \mathscr{G}$. In fact, if $R \in \mathscr{R}$, then $k(R)$ is $\left(G_{k}\right)_{l}$ - stable and so $k(R)=k(G / G(R))$. For every $f \in R,\left(G_{k}\right)_{l} f$ generates a finite dimensional $k$-vector space, Hence there exists a finitely generated over $k\left(G_{k}\right)_{l}$ - stable subring $R_{0}$ of $R$ such that $k\left(R_{0}\right)=k(R)$. Let $W$ denote

${ }^{6}$ C.f. $[1]$ p. 324 . 
the affine variety that has $R_{0}$ as its coordinate ring. One can define a structure of a $G$-homogeneaus space on $W$, since $K\left[R_{0}\right]$ is $G_{l}$ - stable. Let $\eta$ be the canonical mapping of $G / G(R)$ into $W$. Then $\eta$ commutes with the action of $G$ and is birational. Hence $\eta$ is an isomorphism of $G / G(R)$ onto an open subset $\eta(G / G(R))$ of $W$.

Moreover, $R=k[G] \cap k(G / G(R))$, since $R \in \mathscr{R}$ and $k(R)=k(G / G(R))$. This completes the proof of the theorem.

Added in Proof. The equivalence (1) $\Longleftrightarrow(2)$ of Theorem 1 in the case where $k$ is algebraically closed has been proved by E. Abe and T. Kanno (Tohoku Math. Jour. 2nd series 11 (1959), 376-384).

\section{REFERENCES}

1. P. Cartier, Dualité de Tannaka des graoups et des algèbres de Lie, Comptes Rendus de l'Académie des Sciences, Paris, 242 (1956), 322-325

2. C. Chevalley, Fondements de la Géometrie Algebrique, Paris, L'Ecole Normale Superieure, 1958.

3. G. Hochschild and G. D. Mostow, Representations and representative functions of Lie groups, Annals of Math., 66 (1957), 495-542.

4. M. Rosenlicht, $A$ note on derivations and differentials on algebraic varieties, Portugaliae Mathematica, 16 (1957), 43-55.

5. A. Weil, On algebraic groups and homogeneous spaces, Amer. J. Math., 77 (1955), 493512.

University of California and Polish ACAdemy of Sciences 



\section{PACIFIC JOURNAL OF MATHEMATICS}

\section{EDITORS}

\author{
RaLPh S. Phillips \\ Stanford University \\ Stanford, California \\ F. H. BRowNELL \\ University of Washington \\ Seattle 5 , Washington
}

A. L. Whiteman

University of Southern California

Los Angeles 7, California

L. J. Paige

University of California

Los Angeles 24, California

\author{
E. F. BECKENBACH \\ T. M. CHERRY
}

\author{
ASSOCIATE EDITORS

$\begin{array}{lll}\text { D. DERRY } & \text { H. L. ROYDEN } & \text { E. G. STRAUS } \\ \text { M. OHTSUKA } & \text { E. SPANIER } & \text { F. WOLF }\end{array}$

\section{SUPPORTING INSTITUTIONS}

\author{
UNIVERSITY OF BRITISH COLUMBIA \\ CALIFORNIA INSTITUTE OF TECHNOLOGY \\ UNIVERSITY OF CALIFORNIA \\ MONTANA STATE UNIVERSITY \\ UNIVERSITY OF NEVADA \\ NEW MEXICO STATE UNIVERSITY \\ OREGON STATE COLLEGE \\ UNIVERSITY OF OREGON \\ OSAKA UNIVERSITY \\ UNIVERSITY OF SOUTHERN CALIFORNIA
}

\author{
STANFORD UNIVERSITY \\ UNIVERSITY OF TOKYO \\ UNIVERSITY OF UTAH \\ WASHINGTON STATE COLLEGE \\ UNIVERSITY OF WASHINGTON \\ AMERICAN MATHEMATICAL SOCIETY \\ CALIFORNIA RESEARCH CORPORATION \\ HUGHES AIRCRAFT COMPANY \\ SPACE TECHNOLOGY LABORATORIES \\ NAVAL ORDNANCE TEST STATION
}

Mathematical papers intended for publication in the Pacific Journal of Mathematics should be typewritten (double spaced), and the author should keep a complete copy. Manuscripts may be sent to any one of the four editors. All other communications to the editors should be addressed to the managing editor, L. J. Paige at the University of California, Los Angeles 24, California.

50 reprints per author of each article are furnished free of charge; additional copies may be obtained at cost in multiples of 50 .

The Pacific Journal of Mathematics is published quarterly, in March, June, September, and December. The price per volume (4 numbers) is $\$ 12.00$; single issues, $\$ 3.50$. Back numbers are available. Special price to individual faculty members of supporting institutions and to individual members of the American Mathematical Society: $\$ 4.00$ per volume; single issues, $\$ 1.25$.

Subscriptions, orders for back numbers, and changes of address should be sent to Pacific Journal of Mathematics, 103 Highland Boulevard, Berkeley 8, California.

Printed at Kokusai Bunken Insatsusha (International Academic Printing Co., Ltd.), No. 6, 2-chome, Fujimi-cho, Chiyoda-ku, Tokyo, Japan.

\section{PUBLISHED BY PACIFIC JOURNAL OF MATHEMATICS, A NON-PROFIT CORPORATION}

The Supporting Institutions listed above contribute to the cost of publication of this Journal, but they are not owners or publishers and have no responsibility for its content or policies.

Reprinted 1966 in the United States of America 


\section{Pacific Journal of Mathematics}

\section{Vol. 11, No. 4}

A. V. Balakrishnan, Prediction theory for Markoff processes . . . . . . . . . . 1171

Dallas O. Banks, Upper bounds for the eigenvalues of some vibrating systems . . . . 1183

A. Białynicki-Birula, On the field of rational functions of algebraic groups ...... 1205

Thomas Andrew Brown, Simple paths on convex polyhedra .............. 1211

L. Carlitz, Some congruences for the Bell polynomials . . . . . . . . . . . . 1215

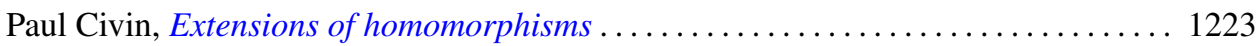

Paul Joseph Cohen and Milton Lees, Asymptotic decay of solutions of differential

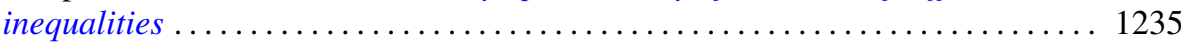

István Fáry, Self-intersection of a sphere on a complex quadric . . . . . . . . . . 1251

Walter Feit and John Griggs Thompson, Groups which have a faithful representation

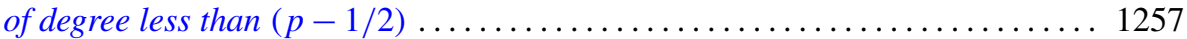

William James Firey, Mean cross-section measures of harmonic means of convex

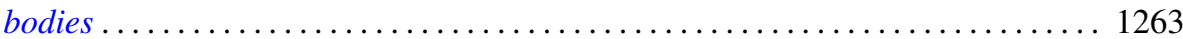

Avner Friedman, The wave equation for differential forms . . . . . . . . . . 1267

Bernard Russel Gelbaum and Jesus Gil De Lamadrid, Bases of tensor products of

Banach spaces ................................... 1281

Ronald Kay Getoor, Infinitely divisible probabilities on the hyperbolic plane . . . . 1287

Basil Gordon, Sequences in groups with distinct partial products . . . . . . . . . . . . 1309

Magnus R. Hestenes, Relative self-adjoint operators in Hilbert space . . . . . . . . . 1315

Fu Cheng Hsiang, On a theorem of Fejér ......................... 1359

John McCormick Irwin and Elbert A. Walker, On N-high subgroups of Abelian

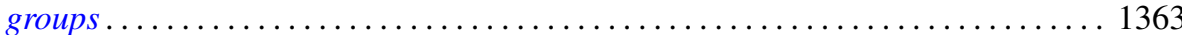

John McCormick Irwin, High subgroups of Abelian torsion groups . . . . . . . . . 1375

R. E. Johnson, Quotient rings of rings with zero singular ideal . . . . . . . . . . . 1385

David G. Kendall and John Leonard Mott, The asymptotic distribution of the time-to-escape for comets strongly bound to the solar system ...

Kurt Kreith, The spectrum of singular self-adjoint elliptic operators ....

Lionello Lombardi, The semicontinuity of the most general integral of the calculus of variations in non-parametric form ................................

Albert W. Marshall and Ingram Olkin, Game theoretic proof that Chebyshev inequalities are sharp

Wallace Smith Martindale, III, Primitive algebras with involution . . William H. Mills, Decomposition of holomorphs ..............

James Donald Monk, On the representation theory for cylindric algebras . . . . . . 1447

Shu-Teh Chen Moy, A note on generalizations of Shannon-McMillan theorem . . . . 1459

Donald Earl Myers, An imbedding space for Schwartz distributions . .

John R. Myhill, Category methods in recursion theory .........

Paul Adrian Nickel, On extremal properties for annular radial and circular slit mappings of bordered Riemann surfaces

Edward Scott O'Keefe, Primal clusters of two-element algebras . .

Nelson Onuchic, Applications of the topological method of Wazewski to certain

problems of asymptotic behavior in ordinary differential equations ...

Peter Perkins, A theorem on regular matrices................

Clinton M. Petty, Centroid surfaces .... 